J. Lake Sci. (湖泊科学) , 2011, 23(4): 549-554

http: //www. jlakes. org. E-mail : jlakes@niglas.ac.cn

(c) 2011 by Journal of Lake Sciences

\title{
太湖梅梁湾摇蚊亚化石沉积记录及营养演化研究”
}

\author{
曹艳敏 ${ }^{1,2}$, 张恩楼 ${ }^{1 * *}$, 沈 吉 ${ }^{1}$, 刘恩峰 $^{1}$, 陈 旭 ${ }^{1,2}$ \\ ( 1 : 中国科学院南京地理与湖泊研究所湖泊与环境国家重点实验室,南京 210008) \\ (2:中国科学院研究生院, 北京 100049)
}

\begin{abstract}
摘 要: 对太湖梅梁湾 T0905 岩芯摇蚊幼虫亚化石组合进行了分析, 探讨了自 1940 年以来梅梁湾湖区摇蚊幼虫对营养 盐演化的响应. 结果表明,梅梁湾湖区摇蚊组合变化以 1970 年为分界点,经历了由 Tanytarsus 为优势属种向富营养属种 Chironomus plumosus-type 和 Microchironomus 为优势组合转变的过程. 1970 年以前,沉积物中摇蚊以 Tanytarsus 为主, 梅梁 湾湖区水质相对较好, 为中营养水平; 1970 年以来, Tanytarsus 的相对丰度伴随 Chironomus plumosus-type 的增加而迅速降 低, 耐营养种 Propsilocerus akamusi-type 也开始出现, 尤其是 1990s 以来, 富营养种 Microchironomus tabarui-type 的百分含量 也迅速增加, 梅梁湾湖区营养水平迅速升高, 水体由中富营养水平转变为富营养水平.
\end{abstract}

关键词: 太湖;梅梁湾;摇蚊;沉积记录;富营养化

\section{Subfossil chironomid assemblages in sediments of Meiliang Bay, Lake Taihu and the trophic reconstruction}

CAO Yanmin ${ }^{1,2}$, ZHANG Enlou ${ }^{1}$, SHEN Ji ${ }^{1}$, LIU Enfeng ${ }^{1} \&$ CHEN Xu ${ }^{1,2}$

(1: State Key Laboratory of Lake Science and Environment, Nanjing Institute of Geography and Limnology, Chinese Academy of Sciences, Nanjing 210008, P. R. China)

(2: Graduate School of Chinese Academy of Sciences, Beijing 100049, P. R. China)

\begin{abstract}
Based on chironomid analysis for sediment core T0905 from Meiliang Bay, Lake Taihu, response patterns of chironomid assemblages to lake trophic changes were discussed. Results show that chironomid assemblages experienced a notable community shift from predominant Tanytarsus to Chironomus plumosus-type and Microchironomus dominated at around 1970. Before 1970, chironomid assemblages were dominated by Tanytarsus, which is an indicator of relatively good water quality and moderate trophic status. Subsequently, the relative abundance of Tanytarsus decreased along with the increase of eutrophic indicators such as Chironomus plumosus-type and Propsilocerus akamusi-type. Especially from 1990, the abundance of Microchironomus tabarui-type had a sharp increase, denoting that Meiliang Bay has already become eutrophic level from meso-eutrophication.
\end{abstract}

Keywords: Lake Taihu; Meiliang Bay; chironomids; sediment record; eutrophication

湖泊沉积记录可以作为过去环境变化以及人类活动引起的流域环境变化、湖泊富营养化和酸化的档 案 ${ }^{[1]}$. 其中, 湖泊沉积物中的生物指标是提供古湖沼环境重建最详尽信息的来源之一 ${ }^{[2]}$. 摇蚁 (Diptera: Chironomidae) 幼虫由于发育了几丁质的头囊, 能在沉积物中得以良好保存. 基于世界不同地区摇蚊一湖泊环境 指标转换函数的建立, 摇蚊幼虫亚化石已被成功地应用于各种湖泊环境要素的定量重 ${ }^{[1,3-7]}$. 我国也已在 青藏高原及长江中下游地区分别建立了摇蚁-湖水盐度、摇蚊-湖水总磷转换函数 ${ }^{[8-9]}$, 并在青藏高原苏干湖 盐度重建、武山湖湖水总磷重建以及太白湖营养演化过程研究中得以成功运用 ${ }^{[10-12]}$, 表明摇蚊亚化石是一 种良好的湖水环境指示器,在我国湖泊水环境演化研究中具有非常重要的作用 ${ }^{[12]}$.

太湖是我国第三大淡水湖泊,湖泊面积 $2427.8 \mathrm{~km}^{2}$, 平均水深约 $1.9 \mathrm{~m}$. 近年来, 快速发展的工农业和城

* 国家重点基础研究发展规划项目 (2008CB418103) 资助. 2010-09-08 收稿;2010-11-02 收修改稿. 曹艳敏, 女, 1986 年生, 博士研究生; E-mail :ymcao720@ gmail. com.

** 通讯作者;E-mail: elzhang@ nighas. ac. cn. 
市化进程使得太湖水环境污染日益严重, 富营养化问题愈演愈烈, 太湖水体生态系统遭到严重破坏, 1980s 开始浮游生物多样性减少, 90 年代后每年夏季都有蓝藻暴发 ${ }^{[13]}$. 尤其是毗邻无锡市的梅梁湾湖区, 富营养 化问题非常突出. 由于缺乏长期的监测资料, 该湖区历史时期的湖泊营养演化过程尚不清楚. 尽管近年来许 多古湖沼学工作已经开展 ${ }^{[14]}$, 利用生物指标对该湖营养演化历史的研究仅限于对硅藻和枝角类微化石的探 讨 $^{[15-17]}$, 本文从湖泊古生态学角度出发, 通过对沉积钻孔摇蚊幼虫亚化石组合进行分析, 探讨 1940 年以来 梅梁湾湖区摇蚊属种组合特征及其指示的湖泊营养盐演化过程.

\section{1 材料与研究方法}

\section{1 样品采集}

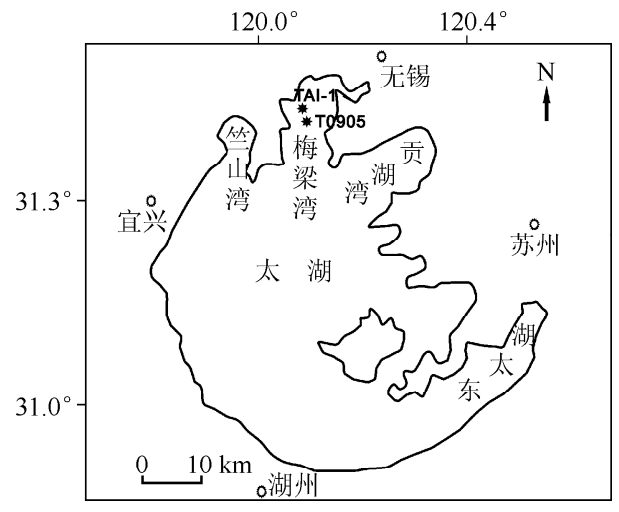

图 1 太湖及采样点位置

Fig. 1 Lake Taihu and sampling sites

2009 年在太湖北部梅梁湾湖区 $\left(31^{\circ} 48^{\prime} \mathrm{N}, 120^{\circ} 17^{\prime} \mathrm{E}\right.$; 图 1) 利用重力采样器采集了长 $50 \mathrm{~cm}$ 的沉积岩芯 ( T0905), 现场以 $1 \mathrm{~cm}$ 间隔分样, 获得的样品密封于塑料袋 内, 带回实验室 $4^{\circ} \mathrm{C}$ 冷藏保存以备分析.

\section{2 实验室分析}

本文所涉及的实验室分析项目包括摇蚊分析, 碳球粒 (Spheroidal Carbonaceous Particles, SCP) 的鉴定和 $\mathrm{Cu} 、 \mathrm{Zn}$ 等元素测定, SCP 针对 $35 \mathrm{~cm}$ 以上层段岩芯, 其它指标对 $30 \mathrm{~cm}$ 以上层段岩芯进行.

摇蚊亚化石分析按照标准实验方法 ${ }^{[18]}$ 进行: 将样品加 人 $10 \% \mathrm{KOH}, 75^{\circ} \mathrm{C}$ 水浴加热 15 分钟后, 依次过 $212 \mu \mathrm{m}$ 和 $90 \mu \mathrm{m}$ 笁, 将样品反洗至烧杯中, 在 25 倍体式显微镜下将摇 蚊头壳化石手工拣出, 用 Hydromatrix ${ }^{\circledR}$ 将其封片, 后在 $100-$ 400 倍生物显微镜下根据文献 [19-22]完成摇蚊属种鉴定. 每个沉积样品中摇蚊幼虫亚化石统计数至少达 50 个壳体. 利用 TILIA 2.0 b. 5, TILIAGRAPH 2. 0 b. 5 软件 ${ }^{[23]}$ 完成摇蚊属种百分比图谱的绘制,并在 CONISS 聚类分析 ${ }^{[24]}$ 的基础上进行摇蚊组合带的划分.

SCP 处理方法依据文献 $[25]$ 进行. 取干样 $0.1-0.2 \mathrm{~g}$, 置于 $12 \mathrm{ml}$ 聚丙烯管中, 依次加人 $3 \mathrm{ml}$ 硝酸、氢氟 酸、盐酸, 分别 $80^{\circ} \mathrm{C}$ 水浴 $2 \mathrm{~h}$, 以去除样品中的有机质、硅酸盐和碳酸盐, 加人蒸馏水离心移取上层清液后, 将 剩余物制成玻片在 400 倍生物显微镜下鉴定、统计大于 $20 \mu \mathrm{m}$ 的 SCP, 最后计算沉积物中 SCP 的浓度, 记为 单位重量沉积物中 SCP 的颗粒数, 以 count/g 来表示.

用于 $\mathrm{Cu} 、 \mathrm{Zn}$ 等元素含量分析的样品低温烘干,研磨至 100 目,采用盐酸-硝酸-氢氟酸消化法处理后,利 用美国 LEEMAN LABS PROFILE 型电感耦合等离子体原子发射光谱仪( ICP-AES)测定.

\section{3 数理统计分析}

摇蚊幼虫亚化石组合受多种环境因子的影响,但并非所有的环境指标都能够与摇蚊组合建立显著的函 数关系 ${ }^{[10,26]}$. 降维对应分析 (DCA) 用于摇蚊亚化石数据分析, 提取影响摇蚊组合分布的主要环境变量, 这是 一种间接梯度分析方法, 以测试主要坐标轴的潜在环境意义, 为主要环境指标的揭示提供依据 ${ }^{[27]}$. 选择至少 在两个或两个以上样品中出现, 含量至少在一个样品中超过 $2 \%$ 的常见属种用于 DCA 分析, 摇蚊幼虫亚化 石含量用平方根转换, 对含量极少的属种进行降权处理, 以减少对结果造成的偏差. DCA 数值分析在 CANOCO version 4.5 软件中进行 ${ }^{[28]}$.

\section{2 研究结果}

\section{1 钻孔年代序列}

本钻孔采用 SCP 区域性事件定年的方法,并与 Rose 等对太湖梅梁湾 TAI-1L 钻孔 (图 1 ) 的分析结果 ${ }^{[29]}$ 进行对比. 煤和石油等化石燃料在 $1750^{\circ} \mathrm{C}$ 的高温下燃烧, 会产生一些由单质碳所组成的多孔性球状颗粒 (SCP), 它伴随发电工业而产生, 随烟尘在大气中扩散, 通过干沉降和降水在沉积物中积累下来, 因而能很好 
地反映区域工业发展历史,记录人类活动对区域环境影响的变化过程. SCP 化学性质相当稳定,能在湖泊沉 积物中得以良好保存, 将区域工业发展记录与 SCP 含量峰值相结合, 可以作为近代沉积物定年很好的 指标 ${ }^{[30-31]}$.

本钻孔 $21 \mathrm{~cm}$ 以下 $\mathrm{SCP}$ 浓度较低 (图 2a), 而后开始迅速增加, 在 $11 \mathrm{~cm}$ 处出现明显的峰值; $21 \mathrm{~cm}$ 以下层 段 $\mathrm{Zn} 、 \mathrm{Cu}$ 含量较低 (分别为 $60 \mu \mathrm{g} / \mathrm{g}, 17 \mu \mathrm{g} / \mathrm{g}$ 左右, 图 2c,2e), Rose 等的研究 (图 2d,2f) 中 $25 \mathrm{~cm}$ 以下 $\mathrm{Zn}$ 的含 量小于 $60 \mu \mathrm{g} / \mathrm{g}$, 而 $\mathrm{Cu}$ 则为 $15 \mu \mathrm{g} / \mathrm{g}$ 左右. 对比 T0905 与 TAI-1L 两岩芯的结果发现, SCP、Zn、Cu 具有相似的 变化规律, 两岩芯中的 $\mathrm{Zn} 、 \mathrm{Cu}$ 含量分别在 $21 \mathrm{~cm} 、 25 \mathrm{~cm}$ 之后呈增加趋势, 同时 $\mathrm{SCP}$ 浓度也分别在约 $21 \mathrm{~cm}$ 、 $25 \mathrm{~cm}$ 后迅速上升,据吴艳宏等研究发现, 1952 年后我国电力工业开始逐年成倍发展, 现今华东电网的主力 发电厂于 1956 年在淮南建成 ${ }^{[31]}$, 之后江苏省的发电量也开始急速增长. 结合 TAI-1L 的测年结果, 可以推断 T0905 钻孔中 $21 \mathrm{~cm}$ 处约为 1955 年. 结合 T0905 沉积柱中 SCP 出现的明显峰值, 与 Rose 等的结果 ${ }^{[29]}$ (图 2b) 对比可知本钻孔中 $11 \mathrm{~cm}$ 处约为 1990 年, 由此推算 1955 到 1990 年间, 梅梁湾湖区平均沉积速率为 $0.31 \mathrm{~cm} / \mathrm{a}$, 而在 1990 至 1998 年间则迅速上升为 $0.75 \mathrm{~cm} / \mathrm{a}$. 依据 1955 至 1990 年及 1990 年以来的平均沉积速率, 大致可 以建立岩芯 T0905 中摇蚊组合发生明显变化的各阶段所对应的年代 (图 3 ).

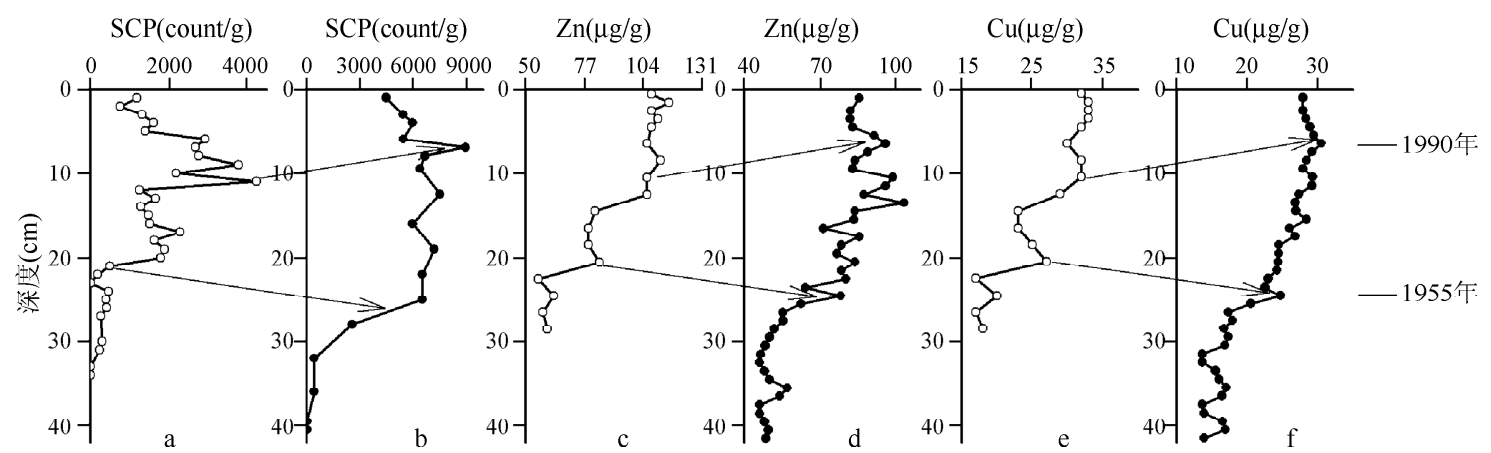

图 2 T0905 (a,c,e) 与 TAI-1L ( b, d,f) 钻孔中 SCP 浓度及 $\mathrm{Zn} 、 \mathrm{Cu}$ 元素变化曲线对比 Fig. 2 Comparison of SCP, Zn and Cu between the two cores:T0905(a,c,e) and TAI-1L(b,d,f)

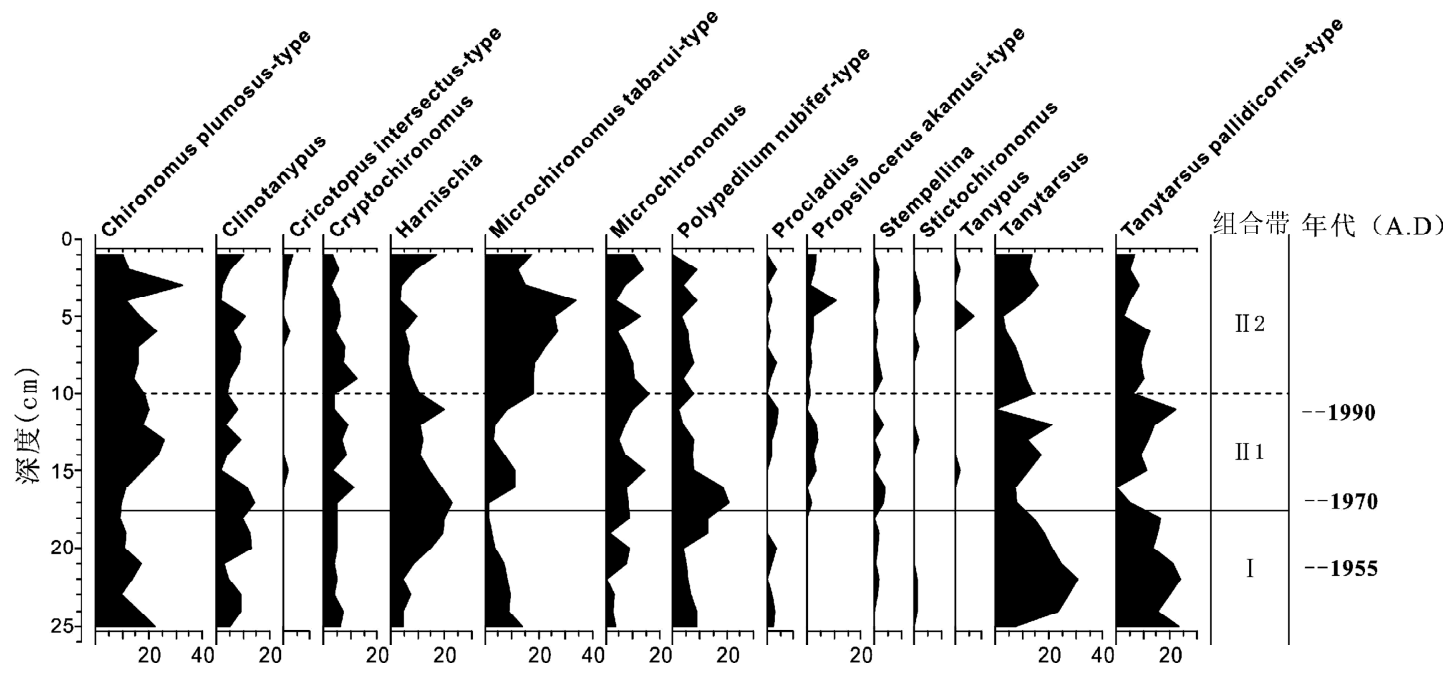

图 3 梅梁湾钻孔 T0905 主要摇蚊属种组合图谱

Fig. 3 Diagram of main chironomid taxa in core T0905 


\section{2 摇蚊亚化石组合}

T0905 孔摇蚊组合分析主要针对钻孔 0-25cm 段进行. 鉴定共发现 24 属 30 种摇蚊, 其中以 Chironomus plumosus-type、Microchironomus tabarui-type、Harnischia、Polypedilum nubifer-type 和 Tanytarsus 为主. 根据 CONISS 聚类分析, 可以将摇蚊属种划分为两个主要的组合带, 其中第二带又可以划分为两个亚带(图 3).

组合带 I : 25-17.5 cm ( 1945-1969 年), 该阶段 Tanytarsus 在整个沉积柱中的百分含量最高 (近 $40 \%$ ), 其中 Tanytarsus pallidicornis-type 的百分含量大于 $20 \%$, 而 Microchironomus tabarui-type、Chironomus plumosus-type、Microchironomus 的丰度相对较低.

组合带 II 1:17.5-10cm (1969-1991 年), 耐营养种 Propsilocerus akamusi-type 在这一阶段经历了从无到 有的过程, Microchironomus 和 Chironomus plumosus-type 的相对丰度逐渐增加, 同时 Tanytarsus 较第一阶段明 显下降, 但 Microchironomus tabarui-type 在这一阶段波动较大, 平均丰度约 6\%, 增加趋势不明显.

组合带 II 2:10-0 cm (1991-2009 年), 这一阶段 Microchironomus tabarui-type 的百分含量较前两阶段有 明显增加 (达 $20 \%$ ), Chironomus plumosus-type 和 Propsilocerus akamusi-type 的丰度也与第二阶段相当, Tanytarsus 却保持了前一阶段的趋势继续减少, 其平均百分含量不到 $18 \%$.

\section{3 数值分析}

摇蚊亚化石数据的 DCA 分析结果表明,第一、二排序轴的特征值较大, 分别解释了 $24.1 \% 、 11.9 \%$ 的摇 蚊亚化石属种组合变率. 在 DCA 属种分布图 (图 4a) 中, 富营养种 Chironomus plumosus-type, Microchironomus tabarui-type, Tanypus, Propsilocerus akamusi-type 分布在图的左侧, 而中营养的 Tanytarsus, Polypedilum nubifer-type 等出现在第一轴的右侧. 在样点分布图上 (图 4b), 第一轴很好的将组合带 I $(18-25 \mathrm{~cm}$ ) 和组合带 II $1(11-17 \mathrm{~cm})$ 、组合亚带 II 1 和组合亚带 II $2(1-10 \mathrm{~cm})$ 区分开来. 可见第一轴代表了一个相对最为重要的 环境变量,显著地影响了摇蚊亚化石组合状况.
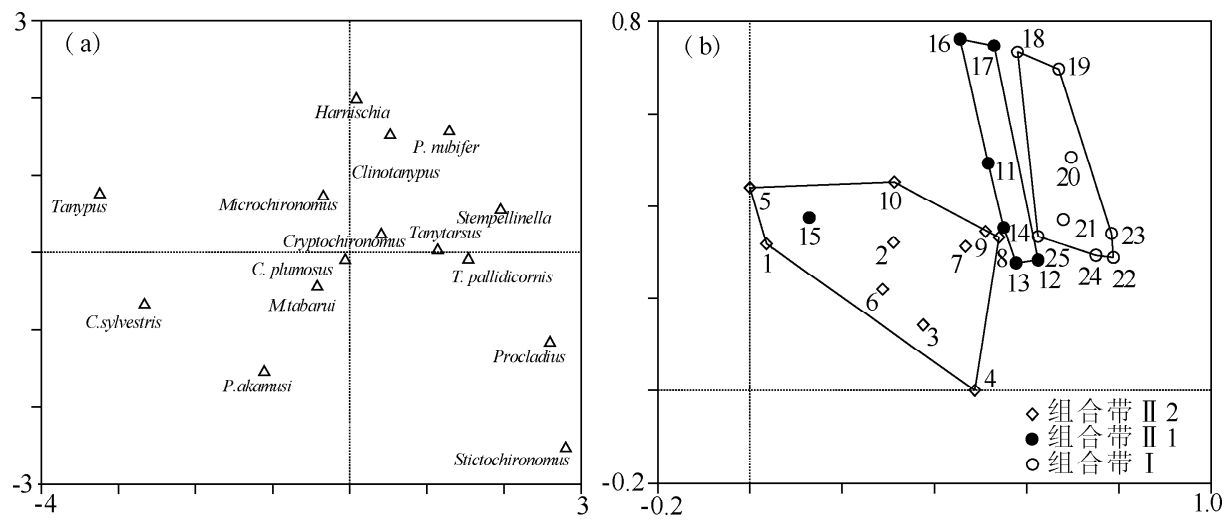

图 4 T0905 钻孔摇蚊属种 DCA 排序分析结果

Fig. 4 DCA ordination results of chironomid data from core T0905

\section{3 讨论}

摇蚊种属的分布受多种环境因子共同影响. 其中温度通过对摇蚊生理机能的显著影响而成为大的地理 尺度上摇蚊分布和属种组合的最重要的影响因子 ${ }^{[18]} \cdot \mathrm{pH}$ 的变化往往能使摇蚊的丰度及多样性降低, 并以多 种方式影响摇蚊种属组合. 与湖泊营养状况直接相关的溶解氧含量可以直接作用于摇蚊的新陈代谢过程, 从而对摇蚊的分布产生影响. 取食状况 (尤其是氮含量) 影响摇蚊幼虫生长速率, 而干旱地区湖泊盐度的含 量则在很大程度上决定了摇蚊分布的格局 ${ }^{[22]}$. 浅水湖泊中, 湖水营养状况的变化通过一系列的生态系统过程 显著地影响摇蚊组合, 因而摇蚊组合的变化能准确反应湖水营养状况的变化 ${ }^{[5]}$. 据张恩楼等的研究, 湖水 TP 等营养指标是影响长江中下游地区湖泊摇蚊幼虫亚化石组合的主要环境因子, Chironomus plumosus-type, Microchironomus tabarui-type, Tanypus, Propsilocerus akamusi-type 在长江中下游地区湖泊摇蚊一湖水总磷转函数中的 
总磷最适值分别为 $1.96,2.25,2.12,2.06$, 高于其他属种 ${ }^{[9]}$, 其他地区的许多研究也说明它们适于在营养程度 较高的湖泊中生存, 为耐营养种 ${ }^{[5,22,32]}$. 这些属种分布于 DCA 属种分布图的左侧, 在 1970 尤其是 1990 年后的 沉积岩芯中显著增加, 而中等营养水平的 Tanytarsus 的相对含量则相应减少, 说明第一轴代表的是营养因子这 一重要环境指标. 样点分布图 (图 4b) 中的各组合单元则分别说明岩芯所代表时期湖泊的营养状态.

摇蚊组合记录的湖水营养状况在 1970 年附近发生明显变化. Microchironomus tabarui-type、Chironomus plumosus-type 和 Tanytarsus 在整个沉积柱中都处于主导地位, 但在 1970 年以前, 前两者的百分含量明显低于 后者. Chironomus plumosus-type 和 Microchironomus tabarui-type 都是湖泊营养化的良好指示者 ${ }^{[5,32]}$. Tanytarsus 对湖泊营养水平的最适度相对较低,一般指示生产力较低的湖泊 ${ }^{[9]}$, 据此推断梅梁湾湖区在这段时期内湖 水状况相对较好, 应为中等营养水平. 1970-1990 年间, Chironomus plumosus-type 迅速增加, 富营养种 Propsilocerus akamusi-type 也开始出现, 这段时期内湖区人类活动不断加强, 围网养鱼, 流域内工业化和城镇化开始 迅速发展, 大量的工农业废水和城镇生活污水直接人湖, 使得湖泊营养负荷进一步增加, 湖泊水体状况日益 下降,此阶段为中-富营养向富营养水平转变的过渡时期. 近 20 年来, 湖泊中上述属种丰度变化不大, 但指 示富营养化水平的 Microchironomus tabarui-type 却在摇蚊属种组合中占据主导优势, 表明湖泊富营养化程度 进一步加重. 本研究中摇蚊亚化石组合反映的湖泊营养状态变化与利用硅藻重建的湖水总磷变化 ${ }^{[15]}$ 及太湖 迅速富营养化的开始 ${ }^{[32]}$ 等研究的结论相吻合.

1970 年以前 Chironomus plumosus-type 等富营养种的存在说明梅梁湾湖区已经历了一个长期的营养程 度较高的过程, 这可能是由于长江流域在历史时期就是人口密集区, 农业生产较为发达, 早期的人类活动可 能导致湖泊营养水平较高,除了生活废水等污染之外,农业的发展必然造成土地利用状况发生变化,土壤侵 蚀也加速了人湖营养物质的供应 ${ }^{[15]}$. 研究表明,太湖湿地和滨海区自 1950s 开始被大量用于水稻种植和养 殖业, 在 $1970 \mathrm{~s}$ 达到高峰 ${ }^{[17]} .1960 \mathrm{~s}$ 后, 无锡等沿湖工业化、城镇化发展迅速, 大量未经处理的工业废水和城 镇生活污水直接人湖, 高含量的总磷、总氮及有机污染物更加剧了太湖的富营养化进程. 为了遏制太湖生态 环境的继续恶化,近年来采取了一系列改善水体水质的措施, 使得补给河流中的 P 含量有所下降 ${ }^{[29]}$,但湖泊 营养程度依然很高, 富营养化现象并未得到有效改善 ${ }^{[33-34]}$, 摇蚊组合中 Microchironomus tabarui-type、Chironomus plumosus-type 耐富营养种的相对丰度 1990s 后不减反增的趋势也说明了这一点.

\section{4 结论}

梅梁湾钻孔中的摇蚊组合显著地响应湖水营养水平的演化. 摇蚊亚化石组合记录说明 1970 年左右为 太湖梅梁湾湖区营养状况演变的转折点, 1970 年以前沉积物中摇蚊以 Tanytarsus 为主, 此时太湖水质相对较 好, 为中等营养水平;1970 至 1990 年间,Tanytarsus 的相对丰度伴随 Chironomus plumosus-type 的减少而迅速增 加, 湖泊营养水平明显较前一阶段增加, 由中一富营养向富营养化转变; 1990 年后, 富营养种 Microchironomus tabarui-type 的百分含量也迅速增加, 湖泊富营养化程度进一步加深, 由中富营养水平转变为富营养水平.

致谢: 袁和忠博士参加野外采样, 夏威岗高级工程师在岩芯年代测定工作中给予很大帮助, 在此一并表示 感谢.

\section{5 参考文献}

[ 1 ] Brodersen KP, Quinlan R. Midges as palaeoindicators of lake productivity, eutrophication and hypolimnrtic oxygen. Quaternary Science Reviews, 2006, 25 : 1995-2012.

[ 2 ] 陈建徽. 我国内陆干旱区过去千年来湿度变化的摇蚊记录及其对比 [学位论文]. 兰州: 兰州大学, 2009.

[ 3 ] Brooks SJ. Fossil midges (Diptera: Chironomidae) as palaeoclimatic indicators for the Eurasian region. Quaternary Science Reviews, 2006, 25 : 1894-1910.

[ 4 ] Eggermont H, Heiri O, Verschuren D. Fossil Chironomidae(Insecta: Diptera) as quantitative indicators of past salinity in African lakes. Quaternary Science Reviews, 2006, 25 : 1966-1994.

[ 5 ] Langdon PG, Ruiz Z, Brodersen KP. Assessing lake eutrophication using chironomids: understanding the nature of community response in different lake types. Freshwater Biology, 2006, 51 : 562-577.

[ 6 ] Heinrichs ML, Walker IR. Fossil midges and palaeosalinity : potential as indicators of hydrological balance and sea-level 
change. Quaternary Science Reviews, 2006, 25: 1948-1965.

[ 7 ] Walker IR, Cwynar LC. Midges and palaeotemperature reconstruction-the North American experience. Quaternary Science Reviews, 2006, 25 : 1911-1925.

[ 8 ] Zhang EL, Jones R, Bedford A et al. A chironomid-based salinity inference model from lakes on the Tibetan Plateau. Journal of Paleolimnology, 2007, 38: 477-491.

[ 9 ] 张恩楼, Bedford A, Jones R 等. 长江中下游地区典型湖泊摇蚊亚化石-湖水总磷定量模型研究. 科学通报,2006,51 (11) :1318-1325.

[10] Zhang EL, Liu EF, Richard J. A 150-year record of recent changes in human activity and eutrophication of Lake Wushan from the middle reach of the Yangze River, China. Journal of Limnology. (待刊).

[11] 张恩楼,曹艳敏,刘恩峰等. 近 150 年来湖北太白湖摇蚊记录与湖泊营养演化. 第四纪研究, 2010,30(6):1-6.

[12] 陈建徽,陈发虎, 张恩楼等. 摇蚊亚化石记录的苏干湖近千年来盐度变化研究. 第四纪研究, 2008,28(2):338-344.

[13］秦伯强,罗潋葱.太湖生态环境演化及其原因分析. 第四纪研究,2004,24(5):561-568.

[14] Wu JL, Huang CM, Zeng HA et al. Sedimentary evidence for recent eutrophication in the northern basin of Lake Taihu, China: human impacts on a large shallow lake. Journal of Paleolimnology, 2007, 38: 13-23.

[15] Dong XH, Bennion H, Battarbee R et al. Tracking eutrophication in Taihu Lake using the diatom record: potential and problems. Journal of Paleolimnology, 2008, 40: 413-429.

[16] 刘桂民,陈非洲,刘正文. 太湖枝角类微化石的初步研究. 湖泊科学, 2008,20(4):470-476.

[17] William YB. 中国太湖 1950 年以来主要环境的变化和迅速富营养化的开始. 古生物学报, 1996,35(2):155-174.

[18] Brooks SJ. Late-glacial fossil midge stratigraphies(Insecta: Diptera: Chironomidae) from the Swiss Alps. Palaeogeography, Palaeoclimatology, Palaeoecology, 2000, 159(3) : 261-279.

[19] Wiederholm T. Chironomidae of the Holarctic region-keys and diagnoses part 1: Larvae. Entomologica Scandinacica, $1983, \mathbf{1 9}($ Suppl) : 1457 .

[20] Oliver DR, Roussel ME. The Insects and Arachnids of Canada. Part 11. The Genera of Larval Midges of Canada. Diptera: Chironomidae. Alaska: Agriculture Canada Publication, 1983, 1746 : 263.

[21] Rieradevall M, Brooks SJ. An identification guide to subfossil Tanypodinae larvae(Insecta: Diptera: Chironomidae) based on cephalic setation. Journal of Paleolimnology, 2001, 25 : 81-99.

[22] Brooks SJ, Langdon PG, Heiri O. The Identification and Use of Palaearctic Chironomidae Larvae in Palaeoecology. QRA Technical Guide No. 10. London: Quaternary Research Association, 2007.

[23] Grimm EC. TILIA version 1. 11. TILIAGRAPH version 1. 18. In Gear, A. (ed. ), A Users Notebook. USA: Illinois State Museum, Springfield, 1991.

[24] Grimm EC. CONISS: A Fortran 77 program for stratigraphically constrained cluster analysis by the method of incremental sum of squares. Computers and Geosciences, 1987, 13: 13-35.

[25] Rose NL. A note on further refinements to a procedure for the extraction of carbonaceous fly-ash particles from sediments. Journal of Paleolimnology, 1994, 11: 201-204.

[26] Langdon PG, Ruiz Z, Wynne S. Ecological influences on larval chironomid communities in shallow lakes: implication for palaeolimnological interpretations. Freshwater Biology, 2010, 55: 531-545.

[27] Ter Braak CJF. Canonical correspondance analysis: A new eigenvector technique for multivariate direct gradient analysis. Ecology, 1986, 67(5) : 1167-1179.

[28] Ter Braak CJF, Smilauer P. CANOCO regerence manual and CanoDraw for Windows user's guide: Software for Canonical Community Ordination( version 4.5). New York: Microcomputer Power, 2002.

[29] Rose NL, Boyle JF, Du Y et al. Appleby P. G. , Bennion H., Cai S. \& Yu L. Sedimentary evidence for changes in the pollution status of Taihu in the Jiangsu region of eastern China. Journal of Paleolimnology, 2004, 32 : 41-51.

[30 ] Rose NL, Harlock S, Appleby PG et al. The dating of recent lake sediments in the United Kingdom and Ireland using spheroidal carbonaceous particle concentration profiles. The Holocene, 1995, 5: 328-335.

[31] 吴艳宏,王苏民,夏威岗等. 近代湖泊沉积物球状碳颗粒 (SCP) 定年. 科学通报,2005,50(7):703-707.

[32] Little JL, Smol JP. Changes in fossil midge (Chironomidae) assemblages in response to cultural activities in a shallow, polymictic lake. Journal of Paleolimnology, 2000, 23: 207-212.

[33] Oin BQ, Xu PZ, Wu QL et al. Environmental issues of Lake Taihu, China. Hydrobiologia, 2007, 581 : 3-14.

[34] 林泽新. 太湖流域水环境变化及缘由分析. 湖泊科学,2002,14(2):111-116. 